(308) Polyxo. Sept. 18. Der eine Anschluss durch Wolken unsicher, weil Planet sehr schwach.

(377) (r893 AN). Sept. I. Bild verwaschen und unruhig, Anschluss sehr unsicher.

München 1897 November.
Die Positionen der Vergleichsterne, welche in den Leipziger AG. Zonen enthalten sind, hat mir Herr Dr. B. Peter gütigst mitgetheilt. Die Ephemeriden von Herrn Prof. Neugebauer verdanke ich der gütigen Mittheilung durch Herrn Prof. R. Luther.

Walter Villiger.

\title{
Einige spectroskopische Beobachtungen mit dem grossen Refractor der Lick-Sternwarte.
}

\section{Von C. Runge.}

Bei einem Besuche der Lick-Sternwarte im September dieses Jahres ist mir die Gelegenheit geboten worden, einige spectroskopische Beobachtungen des Herrn Campbell mit dem grossen Refractor zu wiederholen. Da diese Beobachtungen zum Theil angezweifelt worden sind und bis jetzt durch andere Beobachter nicht haben wiederholt werden können, so ist es vielleicht nicht ohne Interesse, wenn ich beschreibe, was ich gesehen habe.

I) Beobachtung des Orionnebels. Die relativen Intensitäten der Wasserstofflinie $H \beta$ und der Nebellinien sind in verschiedenen Theilen des Nebels sehr verschieden. Wenn der Spalt uber das Trapez gelegt wurde, so schätzte ich die Intensitäten von $H \beta$ und der schwächeren und stärkeren Nebellinie etwa wie $2: 2: 5$. Mit engem Spalt schätzte ich etwas anders, etwa wie $2: 3: 6$. Wurde der Spalt dagegen nach Nordosten vom Trapez in die Nähe des Sterns Bond 734 gelegt, so konnte ich die schwächere Nebellinie überhaupt nicht mehr sehen, während ich die stärkere nur etwa auf $1 / 10$ der Helligkeit von $H \beta$ schätzte. Wurde endlich der Spalt nach Sudwesten etwa eben so weit vom Trapez wie Bond 734 angenommen, so erschien $H \beta$ deutlich stärker als die schwächere der beiden Nebellinien. Bei dieser Beobachtung wurde die stärkere der Nebellinien abgeblendet, damit sie die Schătzung nicht beeinflusste. Diese Beobach. tungen beweisen, dass der Wasserstoff sich weiter verbreitet als der Stoff, der in dem Lichte der beiden Nebellinien leuchtet. Herr Scheiner hat diese Verschiedenheiten in den relativen Intensitäten der drei Linien bestritten und ist sogar so weit gegangen, diese Beobachtung Campbell's als Beispiel anzuführen, woraus dessen Unzuverlässigkeit geschlossen werden könnte (siehe den Schluss seiner Besprechung von Keeler's Arbeit uber die spectroskopische Beobachtung der Nebel in der Vierteljahrsschrift Bd. 32 p. 51 ). Ich kann nur sagen, dass die Beobachtung mit dem grossen Refractor der Lick-Sternwarte ganz leicht war, obgleich der Mond diesen Theil des Himmels mit einigem diffusen Licht erfullte.

2) Wolf-Rayet-Stern, BD. $+30: 3639$. Im Spectrum sind zwei helle Linien zu erkennen, $H \beta$ und eine Linie, deren Wellenlänge Campbell $\mathrm{zu} 5694$ bestimmt hat. Bei richtiger Lage des Spaltes wird 5694 punktförmig, $H \beta$ dagegen kann nicht punktförmig gemacht werden, wie auch der Spalt eingestellt werde. Man kann das continuirliche
Spectrum auf beiden Seiten von $H \beta$ durch richtige Einstellung des Spaltes linienförmig machen, immer ragt $H \beta$ nach beiden Seiten über das Spectrum hinüber. Diese Beobachtung scheint mir keine andere Deutung zuzulassen, als dass der Stern eine Wasserstoffatmosphäre besitzt.

3) BD. - I 2:1 I 72. Mit breitem Spalt sieht man drei Bilder des Nebels in den drei Farben von $H \beta$ und von den beiden Nebellinien. Das Bild in $H \beta$ ist schwächer als das in der stärkeren Nebellinie. Dennoch ist deutlich zu sehen, dass die Gestalt des Nebels in $H \beta$ grösser ist als die Gestalt im Lichte der beiden Nebellinien. Daraus geht hervor, dass der Wasserstoff sich weiter erstreckt als die Partien dès Nebels, die im Lichte der beiden Nebellinien leuchten.

4) $\eta$ Tauri (Alcyone). Im Spectrum von Alcyone war $H \alpha$ deutlich als helle Linie zu sehen, während die Photographien $H \beta$ und die übrigen Wasserstofflinien als dunkle Linien erweisen. Auch diese Beobachtung ist von Herrn Scheiner bestritten worden.

5) Abgesehen von diesem Falle in Spectrum von Alcyone, wo $H \alpha$ eine Emissionslinie, die übrigen Wasserstofflinien dagegen Absorptionslinien sind, habe ich mich gegen meine vorgefasste Meinung überzeugt, dass auch die relativen Intensitäten von $H \alpha, H \beta$ und $H \gamma$ in verschiedenen Spectren sehr verschieden sind. Während ich z. B. in dem Nebel NGC. $7027^{\circ} H \beta$ und $H \gamma$, ganz deutlich, $H \alpha$ aber nicht sehen konnte, war in dem Spectrum von $\gamma$ Cassiopeiae $H \alpha$ sehr deutlich als helle Linie erkennbar, $H \beta$ dagegen sehr viel schwächer, $H \gamma$ gar nicht wabrzunebmen. Das Verhältniss der Intensitäten kehrt sich also gradezu um. In dem einen Fall ist $H \beta$, ja sogar $H \gamma$ stärker als $H \alpha$, in dem andern ist $H \alpha$ stärker als $H \beta$ und $H \gamma$. Die Möglichkeit dieses Intensitätswechsels würde offenbar auch ermöglichen, dass in einem Spectrum $H \alpha$ hell, $H \beta$ und die übrigen Wasserstofflinien dunkel erscheinen. Wir brauchen nur an. zunehmen, dass in der absorbirenden Schicht $H \alpha$ eine geringere Intensität, in der emittirenden dagegen eine grössere Intensität besitzt als die û́hrigen Linien.

6) In dem Nebel NGC. 7027 war auch ausser $H \gamma$, $H \beta$ und den beiden Nebellinien die Linie bei 469 zu sehen, die nach den von Rydberg entdeckten Beziehungen wahrscheinlich zur »Hauptserie « des Wasserstoffs gehört (vergl. Astroph. Journ. Bd. 6, Nr. 3, 1897 ). 